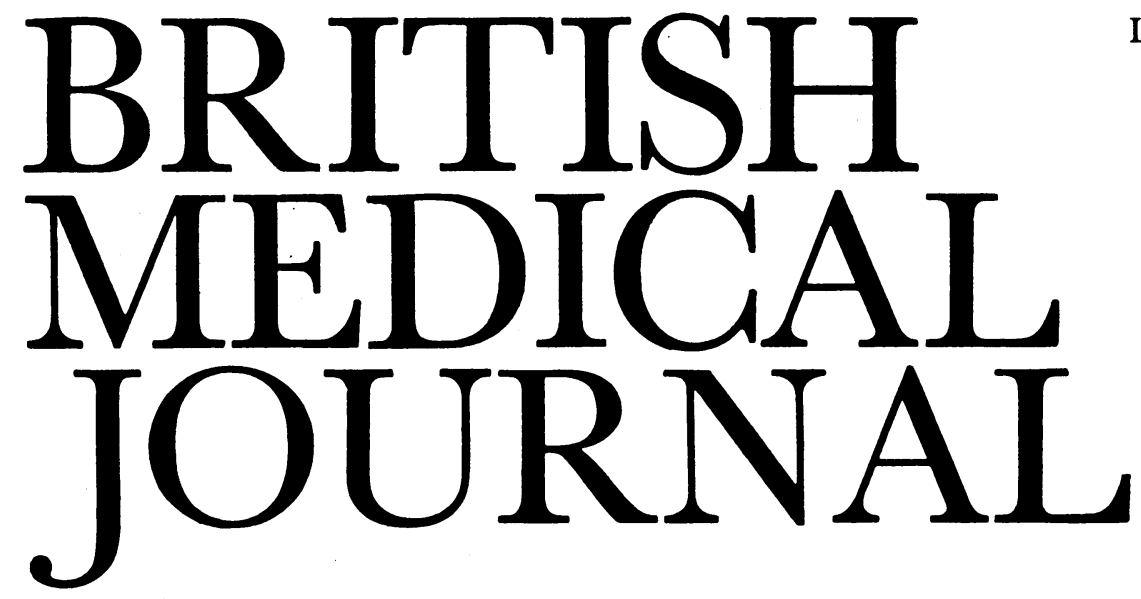

LONDON, SATURDAY 1 FEBRUARY 1986

\title{
Management of depression in general practice
}

Many patients consulting general practitioners show signs of sadness or depression; such patients are at high risk of having psychoactive drugs prescribed. The rise in the volume of anxiolytic and antidepressant drugs prescribed far exceeds any credible rise in the prevalence of disorders appropriately treated by them. So when should depression be treated in a general practice setting? Do recent scientific advances offer the non-specialist useful guidance? Despite exciting developments in the laboratory the answer seems to be "no."

Studies of basic mechanisms have extended our theoretical understanding but not yet influenced practice. ${ }^{1}$ Biological markers or tests such as the dexamethasone suppression test, ${ }^{2}$ the thyroid stimulating hormone test, ${ }^{3}$ and measurement of various urinary metabolites ${ }^{4}$ are valuable research tools, but the results are too unpredictable to be of value to a general practitioner.

Monitoring blood concentrations of antidepressant drugs offers more hope of improving the management of patients. All reports have agreed that no response occurs below a critical concentration, and most have suggested a linear relation between serum concentrations and response. ${ }^{56} \mathrm{With}$ some drugs, notably nortriptyline, there appears to be a "therapeutic window"-a range of concentrations below or above which a response does not occur-and several studies have suggested that high serum concentrations may be negatively correlated with recovery. ${ }^{7.9}$ The observations are still, however, the subject of considerable controversy.

The picture is further complicated by the wide variation known to occur in the serum concentrations achieved by the same dose of antidepressant in different people. ${ }^{10}$ Furthermore, the concentrations are reduced by simultaneous prescription of barbiturates ${ }^{11}$ and raised by concomitant administration of neuroleptics. ${ }^{12}$ The elderly metabolise tricyclic antidepressants more slowly and may achieve therapeutic plasma concentrations with a dose as low as $25-50 \mathrm{mg}$ daily. ${ }^{13}$

Yet while measurement of plasma concentration is unequivocally useful in some circumstances the many sources of error, the expense, and the problems of interpretation suggest that at present such measurements should be undertaken only in specialised units. More careful monitoring and documentation of plasma concentrations in a range of drugs may eventually justify routine use of this approach, but not yet. ${ }^{14}$

What, then, can we offer the primary care physician? The answer is better application of current knowledge and resources.

Sadness is a universal experience and mostly not persistent. Almost always it is related to events, but these life events are often not perceived by the patient and become apparent to the doctor only through sympathetic listening and judicious inquiry. "Well, everyone can master a grief but he that has it," said Shakespeare, ${ }^{15}$ but in clinical practice advice to "put it behind you" or "pull yourself together" is less effective than sympathetic listening. Grief associated with bereavement or other loss will be resolved by mourning and may require some active support from relatives and friends or from professionals. ${ }^{16}$ Other than for very short term relief drugs are not required and may be harmful.

Depression in the "illness" sense implies some disability or impairment which impedes the ability to cope. Here the first essential is accurate diagnosis based on history and mental state examination. When, as is often the case, anxiety and depressive symptoms are mixed an attempt should be made to establish precedence. Where anxiety symptoms arose first, particularly where agoraphobia or more limited phobic avoidance developed before the depression, a simple behavioural approach to the underlying anxiety is often effective. Relaxation training and progressive exposure to feared situations may be achieved with the aid of home training tapes or programmes, self help groups, or referral to a psychologist or psychiatrist.

Occasionally a short course of benzodiazepines may enable the anxiety to be brought under control, but drug treatment should always be associated with supportive counselling including advice on confronting feared situations, and the first step in prescribing these drugs must always be preparing the patient for their withdrawal.

Where panics were an early and prominent symptom hyperventilation should be excluded as an important factor: simple respiratory training may be effective in controlling symptoms. ${ }^{17}$ Where the panics are more persistent and disruptive the American nomenclature now recognises panic disorder with or without phobic avoidance as a distinct 
syndrome. ${ }^{18}$ Those who support this view suggest that tricyclic antidepressants ${ }^{19}$ or the triazalobenzodiazepines such as alprazolan (which are not currently available in Britain) are effective, curative treatments. ${ }^{20}$

When the depression has precedence and is persistent then the previous history, associated life events, and specific symptomatology must be reviewed. Those whose symptoms cluster at the "neurotic" or reactive end of the continuum will tend to show variable disturbance of mood, with clear reactivity and inconsistent diurnal variation, initial insomnia, a search for distraction or comforting, and little by way of biological features. Those whose previous personality is characterised by recurrent episodes of this nature, often associated with gestures of self harm and chaotic social relationships, are unlikely to respond to simple psychotherapy or drug treatment. At some stage an expert assessment is required leading to an agreed treatment or management plan, which will usually rely more heavily on social intervention than psychiatric care or drug treatment.

Where the episode of neurotic depression is a first one or follows a period of good social adjustment drug treatment should be considered-but only if the symptoms are persistent, severe, and not amenable to social intervention and simple counselling. Depression associated with the problems of parenthood, unemployment, poverty, or failure to establish or maintain loving relationships is often relieved more by practical interventions than by formal psychotherapy or drugs. A harassed mother might be enabled to cope by the availability of a washing machine or a babysitter, and many of the socially deprived may benefit from extending their social networks through self help groups, study, or community service.

Simple psychotherapy and counselling do not need highly specialised or esoteric techniques, and most members of the primary care team can develop the skills necessary for therapeutic listening and simple directive counselling or learn where these resources are available to their patients. Some patients, however, may need more specialised therapeutic techniques such as cognitive therapy, but these will probably be beyond the scope of the primary care team..$^{21}$

If the severity or persistence of symptoms seems to justify drug treatment the first decision is between anxiolytic and antidepressant drugs. Where symptoms of tension and initial insomnia predominate a short period of sedation may be helpful, but anxiolytic drugs are not antidepressant and may exacerbate depressive symptoms. Flupenthixol 1-2 mg daily has both antidepressant and anxiolytic effects and is useful in mild cases of depression-but it carries a risk of dyskinesia and should be used only in low dosage and for as short a period as possible.

Treatment with antidepressants, once embarked on, is likely to continue for several months. Doctors do better to be familiar with a small number of drugs than to try out a whole range. Imipramine and amitriptyline are the two standard. antidepressants by which others must be measured, but both have anticholinergic side effects which are troublesome. The sedative properties of amitriptyline may be useful in agitated patients and those with broken sleep or early waking, when a single dose at night can be taken. Treatment with amitriptyline is, however, often associated with a substantial gain in weight and should be used only if such a gain is desirable and acceptable. In obese patients trazodone may be used-it is often associated with loss of weight.

Of the newer antidepressants, mianserin has found favour as having established efficacy, fewer anticholinergic effects, probably less cardiotoxicity, and being safer in overdose. It may cause sedation and gain in weight but can be given as a single nightly dose.

In patients with congestive heart failure, coronary artery disease, a conduction defect, or a recent myocardial infarction antidepressants are best avoided, but if they seem essential mianserin has a larger margin of safety than the tricyclics. ${ }^{22}$ Nomifensine has now been withdrawn from the market in Britain because of protean manifestations possibly attributable to immune mechanisms. ${ }^{22 a}$ Electroconvulsive therapy is safer and has a more rapid effect than drugs.

Where epilepsy or a reduced threshold for fits is a problem desipramine and trazodone or viloxazine are less likely to be associated with fits than other antidepressants (except the monoamine oxidase inhibitors). Monoamine oxidase inhibitors are still useful in some circumstances and may be used in combination with tricyclics. ${ }^{23}$ That combination is, however, sufficiently hazardous to be justified only in the hands of experts.

Having chosen an antidepressant, the clinician will improve the patient's compliance if he warns both of the likely side effects and of the delay of up to two or three weeks before maximum effects are likely to be achieved. A single nocturnal dose also aids compliance, reduces the daytime adverse effects, and aids sleep. The starting dose should be about one third of the standard adult dose, and it may be increased in two stages after intervals of three or four days. Treatment should continue for one month before it can be regarded as having failed. The most common cause of failure is noncompliance and the second is inadequate dosage. The usual maintenance doses suggested in the British National Formulary tend to be on the low side for an average adult and should be increased for anyone of above average size and reduced by half in the frail elderly. If possible a single drug only should be prescribed.

In depression drugs can never be more than half the story; some counselling or support is always necessary. When the patient admits to suicidal fears or ruminations, expresses difficulty in coping with small children, or speaks of unworthiness and hopelessness admission to hospital must be considered. Delusional depression is often uninfluenced by antidepressants but responds well to electroconvulsive therapy. ${ }^{24}$

If an antidepressant in adequate dosage is not producing some improvement within two weeks a change in strategy is usually called for but is probably best undertaken in consultation with an expert. Supplementary lithium, ${ }^{25}$ triiodothyronine, ${ }^{26}$ or oestrogen $\mathrm{s}^{27}$ may increase response to the drug, or it may be advisable to change to a different drug.

Electroconvulsive therapy remains the most rapid and effective treatment of severe depression and is probably used less often than it should be.

Once remission is achieved some continuing medication is required for six months if the risk of relapse is to be reduced. After six months withdrawal should be gradual, over four to eight weeks.

Eventually the laboratory will tell us which patients need which treatment and will monitor its effectiveness. Until those techniques are perfected careful listening and judicious treatment can alleviate most of the misery associated with the common depressive disorders.

SYDNEY BRANDON

Professor of Psychiatry, University of Leicester, Leicester LE2 7LX 
Zis AP, Goodwin FK. The amine hypothesis. In: Paykel ES, ed. Handbook of affective disorders. Edinburgh: Churchill Livingstone, 1982:175-90.

2 Greden JF, Gardner R, King D, Gruhaus L, Carroll J, Kronfol Z. Dexamethazone suppression test in antidepressant treatment of melancholia. Arch Gen Psychiatry 1983;40:493-500.

Kirkegaard C, Bjorum N, Cohn D, Faber J, Lauridsen UB, Nerop J. Studies on the influence of biogenic amines and psychoactive drugs on the prognostic value of the TRH stimulation test in endogenous depression. Psychoneuroendocrinology 1977;2:131-6.

4 Extein I, Gold MS, Pottash ALC. Psychopharmacologic treatment of depression. Psychiatr Clin North Am 1984;7:503-16.

5 Glassman AH, Perel JM, Shostak M, Kantor SJ, Fleiss JL. Clinical implications of imipramine plasma levels for depressive illness. Arch Gen Psychiatry 1977;34:197-204.

Reisby N, Gram LF, Bech P, et al. Imipramine: clinical effects and pharmacokinetic variability. Psychopharmacology 1977;54:263-72.

Asberg $M$, Cronholm B, Siogvist F, Tuck D. Relationship between plasma level and therapeutic effect of nortriptyline. BrMed $\mathcal{F} 1971 ;$ iii:331-4.

Kragh-Sorensen P, Hansen CE, Bastrup PC, et al. Self-inhibiting action of nortriptyline's antidepressive effect at high plasma levels. Psychopharmacologia 1976;45:305-12.
ant

Appelbaum PS, Vasile RG, Orsulak PJ, Schildkraut JJ. Clinical utility of tricyclic antidepressant blood levels: a case report. Am $\mathcal{F}$ Psychiatry 1979;136:339-41.

10 Asberg M. Plasma nortriptyline levels - relationship to clinical effects. Clin Pharmacol Ther 1974;16:215-29.

1 Luscombe DK. Factors influencing plasma drug concentrations. I Int Med Res 1977;5(suppl); 82-97.

12 Nelson JC, Jatlow PI. Neuroleptic effect on desipramine steady-state plasma concentrations. $A m \mathcal{F}$ Psychiatry 1980;137:1232-4.

13 Nies A, Robinson DS, Friedman MJ, et al. Relationship between age and tricyclic antidepressant plasma levels. Am F Psychiatry 1977;134:790-3.
14 American Psychiatric Association Task Force. Tricyclic antidepressants-blood level measurements and clinical outcome: an APA Task Force report. Am J Psychiatry 1985; 142:155-62.

15 Shakespeare W. Much ado about nothing. III.ii.28.

16 Parkes CM. Bereavement: studies of grief in adult life. London: Tavistock, 1972.

17 Clark DM, Salkovis PM, Chalkley AJ. Respiratory control as a treatment for panic attacks. $\mathcal{J}$ Behav Ther Exp Psychiatry 1985;16:23-30.

18 American Psychiatric Association. Diagnostic and statistical manual of mental disorders. 3rd ed Washington: American Psychiatric Association, 1980

19 Leibowitz MR. Imipramine in the treatment of panic disorder and its complications. Psychiat Clin North Am 1985;8:37-48.

20 Gelder M. Cognitive therapy. In: Granville-Grossman K, ed. Recent advances in clinical psychiatry. Edinburgh: Churchill Livingstone, 1985:1-21.

21 Sheehan DV. MAOI's and alprazolam in the treatment of panic disorder and agoraphobia. Psychiatr Clin North Am 1985;8:49-62.

22 Orme ML'E. Antidepressants and heart disease. Br Med f 1984;289:1-2.

22a Anonymous. Trouble with nomifensine. Drug Ther Bull 1985;23:98-100.

23 Pare CMB. The present status of monoamine oxidase inhibitors. Br f Psychiatry 1985; 146:576-84

24 Glassman AH, Roose SP. Delusional depression: a distinct clinical entity? Arch Gen Psychiatry 1981;38:424-7.

25 De Montigny C, Grunberg F, Mayer A, Deschenes JP. Lithium induces rapid relief of depression in tricyclic antidepressant drug non-responders. Br f Psychiatry 1981;138:252-6.

26 Goodwin FK, Prang AJ Jr, Muscettola G, Lipton MA. Potentiation of antidepressant effects by L-triiodothyronine in tricyclic non-responders. Am $\mathcal{F}$ Psychiatry 1982;139:34-8.

27 Klaiber EL, Broverman DM, Vogel W, Kobayashi Y. Estrogen therapy for severe persistent depressions in women. Arch Gen Psychiatry 1979;36:550-4.

\section{A fairer deal from RAWP}

How should the funds of the National Health Service in England be distributed? In 1975 the government set up the Resource Allocation Working Party "to review the arrangements for distributing NHS capital and revenue . . securing, as soon as practicable, a pattern of distribution responsive objectively, equitably and efficiently to relative need." The working party's formula, now known as RAWP, has survived intact through a decade of changes in governments and ministers. ${ }^{1}$

The largest part of NHS expenditure is on hospitals. Before RAWP funds were allocated according to the historical spending by hospitals, with a proportion reserved for new developments. RAWP was designed to reflect health care needs, and the formula's main component is the size of the population to be served. But the low growth of the total NHS budget has meant that RAWP has inevitably penalised health authorities that have many hospitals and declining or static populations (especially in the inner cities) and favoured those with fewer hospitals or growing populations (in the East Midlands and the southern shire counties). Thus, paradoxically, RAWP has come to appear to be "a method of taking away money from poor people in rich areas and giving it to rich people in poor areas."

Commentaries on RAWP in the late 1970s questioned whether it adequately reflected the costs of social deprivation and of medical education and research in teaching hospitals. A Department of Health and Social Security advisory group reviewing these issues was prematurely wound up in 1980 but concluded that the formula could not be refined "until some of the gaps in basic information have been filled." In practice, because of its broad approach towards equity and because hospital services have not and do not vary in response to variations in urban deprivation, RAWP has generally been supported at the regional level. It is subregional RAWP, redistributing resources among districts, that has caused more concern.

The central issue, identified in a new report by the Nuffield Provincial Hospitals Trust, is the funding of acute hospitals. ${ }^{4}$ Should districts be moving towards providing all non-regional acute services themselves, or should districts with an apparent excess of hospital beds and outpatient clinics continue to provide these services for under-resourced districts? The report suggests that building new hospitals will be more expensive than allowing patients to travel to the existing ones. It points to the substantial political difficulties of closing hospitals in poor inner city areas-and it demands protection for the traditional "centres of excellence," especially the London teaching hospitals.

Other criticisms of RAWP have been made by Bevan and colleagues. $^{5.7}$ In inner London, where well resourced hospitals are sited too close to each other, health authority boundaries do not match hospital catchments. Districts lose money under RAWP if their residents go to hospitals outside their district-yet they cannot control these flows. Instead, Bevan proposes that district boundaries should be ignored and that general practitioners and their patients should shop among different hospitals to find short waiting times or underused capacity. Bevan believes that this "simulated market" would be efficient and says that "only totalitarian bureaucrats would want ... to override freedom of choice."

But the NHS does not need markets. ${ }^{8}$ In contrast to insurance systems of financing found in some other countries, the NHS is able to identify budgets that relate directly to specific populations; and strategies that seek to improve health by an appropriate mix of treatment, care, and prevention can readily be evaluated against these budgets. ${ }^{9}$ In a population based service better coordination between hospitals and local community services might allow large shifts of resources from hospital to day and home care, even in the acute specialties, with substantial reduction of costs-a process that is already happening for commercial reasons in the United States. And clearer responsibilities for the resident population will also be welcomed by local authorities, whose cooperation is vital to develop better services for discharged patients and long term care.

Instead of dismantling subregional RAWP we should be thinking how an improved formula could strengthen health authorities and help general managers with their new responsibilities. The most important change would be to remove compensation for cross boundary flows from the 